\title{
Importance of histopathology in diagnosis of unilateral nasal polyps
}

\author{
Rameez Shah", *, Md. Shahriar Islam², Md. Rafiqul Islam³ , Anika Arfin ${ }^{4}$ \\ ${ }^{1}$ ENT Specialist, H=52, L=3, Ibrahim Colony, Hyderpora-190014, Srinagar, Kashmir, India \\ ${ }^{2}$ Dept of Otolaryngology \& Head-Neck Surgery, Dhaka Medical College Hospital, Dhaka-1000, Bangladesh \\ ${ }^{3}$ Diabetic Association Medical College Hospital, Faridpur-7800, Bangladesh \\ ${ }^{4}$ Dept of Internal Medicine, Dhaka Medical College Hospital, Dhaka-1000, Bangladesh
}

\section{Email address:}

rameezshah527@yahoo.com (R. Shah)

\section{To cite this article:}

Rameez Shah, Md. Shahriar Islam, Md. Rafiqul Islam, Anika Arfin. Importance of Histopathology in Diagnosis of Unilateral Nasal Polyps. Clinical Medicine Research. Vol. 3, No. 4, 2014, pp. 87-89. doi: 10.11648/j.cmr.20140304.11

\begin{abstract}
Nasal polyps are one of the most frequent clinical entities encountered by otolaryngologists. However, nasal polyps should be regarded as "signs" and not "diagnosis", unless subject to histopathology-especially when the case is of unilateral nasal polyps, as histopathological evaluation is mandatory to reach a confirmed diagnosis. This study was thus aimed to establish the importance of histopathology in diagnosis of unilateral nasal polyps (as many otolaryngologists still do not agree with the need to do so). A prospective study done in the Dept of Otolaryngology, Dhaka Medical College Hospital, Dhaka, Bangladesh from January'2012 to June'2013 on 69 patients clinically diagnosed as unilateral nasal polyps and surgically treated with subsequent histopathological evaluation. It was found that histopathology was very important and conclusive in the diagnosis of nasal polyps.
\end{abstract}

Keywords: Nasal Polyp, Histopathology

\section{Introduction}

The nose is one of the important organs for physical looks of man and is also an organ through which he perceives the external environment.

It is true that some of the lesions described here are commonly found and some are rare. But it is imperative that the clinician should be aware of these lesions because the eye is blind for what the mind does not know. Nasal polyps remain a significant challenge to the treating physician. The treatment modalities of nasal polyps encompass both medical and surgical modalities following the assessment of the patient.

The word "polyp" which is originally Greek, has undergone latinization and means (polypous) many footed. Nasal polyps are defined as pearly white, painless, prolapsed pedunculated parts of the nasal mucosa. These are unique in position and composition. Small polyps may not produce symptoms. Symptom producing polyps can cause nasal airway obstruction, postnasal drainage, dull headache, snoring, and rhinorrhoea. Associated hyposmia or anosmia is common.

Nasal polyp is applied somewhat loosely to a projection of edematous mucous membrane. Polyp may present in the nasal cavity with a grape like appearance, having a body and a stalk. The polyps protrude in to the nasal cavity from the middle and superior meatus resulting in nasal blockage and abolishing airflow to the olfactory region ${ }^{1}$. Any condition of hyperemia or inflammation within the nasal cavity may give rise to polypus formation.

Though, a large proportion of nasal polyps are generally allergic in origin, these may accompany chronic rhinitis, sinus suppuration, benign or malignant nasal or paranasal sinus disease ${ }^{2}$. Bilateral nasal polyps are usually allergic and clinical diagnosis is certain. But in case of unilateral nasal polyps clinical diagnosis cannot be ascertained unless confirmed by histopathology, and in some cases without histopathology the real diagnosis may never be confirmed.

Nasal polyps are common, affecting up to four percent of the population. Their etiology remains unclear, but they are known to have associations with allergy, asthma, infection, cystic fibrosis, and aspirin sensitivity. Computerized tomography allows evaluation of the extent of the disease and is essential when surgical treatment is to be considered.

There is good evidence for the use of corticosteroids (systemic and topical) both as primary treatment and as 
postoperative prophylaxis against recurrence. Surgical treatment has been refined significantly over the past twenty years with the advent of endoscopic sinus surgery. Symptomatic improvement is more in Endoscopic sinus surgery (ESS) compared to conventional procedures (including polypectomy, Caldwel-luc and intranasal ethmoidectomy).

Conditions such as malignant tumors, inverted papilloma and in a child, meningocoele, all of which may masquerade as simple polyps and may not always be clinically conclusive especially when polyps are unilateral. Unilateral nasal polyps should always be regarded with suspicion and histology is needed in order to exclude malignancy ${ }^{4}$. As there is no general consensus about the importance of routine nasal polyp histology and some even think it as unnecessary when malignancy is not suspected on clinical grounds 5 .

A prospective study was conducted in the Dept of ENT \& Head Neck Surgery, Dhaka Medical College Hospital, Dhaka, Bangladesh from January'2012 to June'2013 to analyze the importance of histopathology in diagnosis of unilateral nasal polyps.

\section{Materials and Methods}

Total 69 patients of both sexes of different age groups (youngest 2 years and oldest 70 years) clinically diagnosed unilateral nasal polyp January'2012 to June'2013 were included in this study. All the patients had surgery for unilateral nasal polyps and specimen sent for histopathological examination.

The study was done at the Dept of ENT \& Head Neck Surgery, Dhaka Medical College Hospital, Dhaka, Bangladesh. The data was collected purposively including age, sex, clinical diagnosis, and histopathological reports. The classification of tumors of nose and paranasal sinuses followed in this study was as described by WHO.

All the data were analyzed by simple statistical methods and written informed consent was taken from the subjects under the study and the study does not serve any conflict of interest to any person who so ever.

\section{Results}

The histopathological diagnosis of 69 patients who had nasal polypectomy operation revealed 61 (88.41\%) were non-neoplastic and $08(11.59 \%)$ were of neoplastic origin. The distribution of histopathological diagnosis is shown in Table-I.

Table I. Unilateral nasal polyps: histopathological results $(n=69)$.

\begin{tabular}{llll}
\hline S. No. & Histopathological Diagnosis & $\begin{array}{l}\text { No. of } \\
\text { cases }\end{array}$ & $\begin{array}{l}\text { Percent } \\
(\%)\end{array}$ \\
\hline 1 & Allergic or inflammatory polyps & 59 & 85.5 \\
2 & Rhinosporidiosis & 2 & 2.9 \\
3 & Neoplastic & 8 & 11.6 \\
& Total & 69 & 100 \\
\hline
\end{tabular}

Among the neoplastic cases $08(11.59 \%)$ of which benign $04(5.80 \%)$ and the rest of the $04(5.80 \%)$ were malignant (Table II).

Table II. Nasal polyp histopathology results neoplastic cases. $(n=08)$

\begin{tabular}{lll}
\hline Histopathological Diagnosis & $\begin{array}{l}\text { No. of } \\
\text { cases }\end{array}$ & $\begin{array}{l}\text { Percent (\%) of } \\
\text { Total (n=69) }\end{array}$ \\
\hline Benign: & & \\
$\begin{array}{l}\text { Epithelial : } \\
\text { Transitional Cell papilloma (Inverted }\end{array}$ & 2 & \\
Papilloma) & 1 & \\
Adenoma & 1 & \\
Mesenchymal : Fibroma & 4 & \\
Subtotal: & & \\
Malignant: & 1 & \\
Squamous cell carcinoma & 1 & \\
Adenoid cystic carcinoma & 2 & \\
Rhabdomyosarcoma & 4 & \\
Sub-total & 8 & 11.6 \\
Total & & \\
\hline
\end{tabular}

The clinical diagnosis did not at all correlate with the pathological diagnosis in 10 cases (i.e. 14.49\%) and there was no clinical suspicion of underlying pathology. (Table III).

Table III. Clinical Diagnosis Vs Histopathological Diagnosis in 10 cases.

\begin{tabular}{lllll}
\hline S. No & Clinical Diagnosis (unilateral) & Age & Sex & Histopathological Diagnosis \\
\hline 1 & Nasal polyp (Lt) & 55 & F & Squamous cell carcinoma \\
2 & Nasal polyp (Rt) & 50 & M & Adenoid cystic carcinoma \\
3 & Antrochonal polyp (Rt) & 6 & M & Rhabdomyosarcoma \\
4 & Antrochonal polyp (Rt) & 4 & M & Rhabdomyosarcoma \\
5 & Nasal polyp (Rt) & 10 & M & Adenoma \\
6 & Nasal polyp (Rt) & 20 & F & Inverted papilloma \\
7 & Nasal polyp (Lt) & 50 & Inverted papilloma \\
8 & Nasal polyp (Rt) & 47 & F & Fibroma \\
9 & Nasal polyp (Rt) & 19 & M & Rhinosporidiosis \\
10 & Nasal polyp (Lt) & 21 & F & Rhinosporidiosis \\
\hline
\end{tabular}

\section{Discussion}

Total sixty nine patients were reviewed whose unilateral nasal polypectomy operations and specimen were examined histopathologically. As only unilateral nasal polyps were sent for histopathological confirmation, the clinical diagnosis and histopathological reports were compared to determine the accuracy of clinician's ability to clinically identify nasal polyps of sinister pathology.

In this series there were $59(85.50 \%)$ histopathology reports 
of nasal polyps with inflammatory/ allergic in origin, 02 $(2.90 \%)$ were rhinosporidiosis and 08(11.59\%) were neoplastic. In about 10 cases (i.e. $14.49 \%$ ) the clinical diagnosis differed from the pathological diagnosis as presented in Table-III. In two cases of clinically suspected unilateral nasal polyp, the histopathological diagnosis was made out inverted papilloma. In one case, adenoid cystic carcinoma was detected histopathologically in a clinically suspected case of nasal polyp. In two cases of antrochoanal polyp the pathological diagnosis was made out rhabdomyosarcoma.

One case of unilateral polyp was diagnosed as moderately differentiated squamous cell carcinoma by pathologist. In one case, unilateral nasal polyp of a10 year old boy was diagnosed adenoma histopathologically.

It is worth noting that as there is no general consensus about the need for routine histopathology of nasal polyps among many otolaryngologists, so the need for such studies is imperative. According to Kale SU et $\mathrm{al}^{6}$, there was $99.7 \%$ correlation between clinical diagnosis and histological diagnosis in a prospective study done the authors seem to be against the practice of sending nasal polyps for routine histological examination.

Alum Jone et $\mathrm{al}^{5}$ pointed out that only $38 \%$ of ENT surgeons take the help of pathologist in confirming their diagnosis in cases of nasal polyposis. The study by Diamantopoulous-II et $\mathrm{al}^{3}$ in 2201 cases justified routine histological examination of nasal polyps.

In this study however, the use of clinical criteria as a method for selecting nasal polyps for histopathology proved inadequate as several cases with sinister pathology would have missed /escaped diagnosis. This finding was studied in respect of inverted papilloma, which revealed that it is difficult to distinguish inflammatory nasal polyps from inverted papilloma ${ }^{7}$ and further noted the importance of having a differential diagnosis between sinonasal chronic infection and sinonasal tumors ${ }^{8}$.

This finding is in agreement with recommendations of other studies that have been reported on various tumors of nasal cavity $^{9}$, vascular turnors ${ }^{10}$, Rhinosporidiosis ${ }^{11}$.

Thus the importance of histopathology in diagnosis of unilateral nasal polyps is inevitable, even though some studies on nasal polyps do suggest certain definite clinical criteria to identify nasal polyps of sinister pathology (like an appearance that is friable, haemorrhagic, indurated, unilateral and a history of pain, radiological evidence of bony erosion or previous abnormal histopathology), but then the role of histopathology in diagnosis of unilateral nasal polyps should seldom be neglected.

\section{Conclusion}

Nasal polyps are common, affecting one to four per cent of the population. Their cause, however, remains unknown and it is possible that it is not the same in all patients. They have a clear association with asthma, aspirin sensitivity and cystic fibrosis. Histologically they demonstrate large quantities of extracellular fluid, mast cell degranulation and an infiltrate of inflammatory cells, usually eosinophils. While this appearance would suggest an allergic pathology there is little conclusive evidence to support this in most patients. There is, however, some preliminary evidence to suggest that a local allergic process could be the cause.

The etiology of nasal polyposis is mainly unknown although it has been connected with many clinical conditions. The long-term clinical course of nasal polyposis is largely unknown, because long-term follow up studies on the recurrence of nasal polyposis have rarely been reported.

All the masses of the nose and PNS should be studied carefully and when surgically removed they should be subjected to histopathological examination not only to confirm diagnosis but also to rule out malignancy.

Based on the observations made in the present study, it is strongly believed that routine histopathological examination is necessary in cases of unilateral nasal polyps and further studies are recommended to strongly ascertain this fact, which would be cumulative to the better patient management, rather than be a contradiction to the clinical eye!

\section{References}

[1] Niels Mygind and Valeri J Lund,Nasal polyposis, Scott-Browns Otorhinolaryngology, Head and Neck surgery. $7^{\text {th }}$ edition, volume $2 \mathrm{~A}$, chapter 121,1549 .

[2] G. Sreenivas,VS Kiranmayi. Histopathology of nasal polyps-a retrospective study; Indian Journal of Otolaryngology and Head and Neck surgery, special issue $2005 ; 65$.

[3] Diamantopoulos II, Jones NS, Lowe. All nasal polyps need histological examination: an audit based appraisal of clinical practice. JLO 2000; 114(10): 755 -9.

[4] Niels Mygind and Valeri J Lund. Nasal polyposis, Scott-Browns Otorhinolaryngology, Head and Neck surgery. $7^{\text {th }}$ edition, volume $2 \mathrm{~A}$, chapter 121,1556 .

[5] Alum-Jones T, Leighton SE., Morrissey MS. Is routine histological examination of nasal polyps justified? Clin Otolaryngol 1990; 15(3): 217 - 9.

[6] Kale SU, Mohite U, Rowlands D, Drake Lee AB. Clinical and histopathlogical correlation of nasal polyps: are there any surprises? Clin.Otolaryngol 2001; 26(4): 321 - 3.

[7] Lawson WH, Saarai CM et.al. Inverted papilloma:A report of 112 cases, Laryngoscope 1995;105: 282 - 288.

[8] Phillips PF, Gustafson RO, Facex. GW. The Clinical behaviour of inverting papilloma of the nose and paranasal sinuses: Report of 112 cases and Review of the literature; Laryngoscope 2000; 100: 463-469.

[9] Lund VJ. Malignant tumors of the nasal cavity and paranasal sinuses. ORL 1983; 46: 1-12.

[10] Osborn DA. Haemangiomas of the nose. JLO 1959; 73: 174 179.

[11] Sathyanarayana C. Rhinosporidiosis with record of 255 cases. Acta Otolaryngol 1960; 51: 348 - 366. 